Clinical management in an evidence vacuum: pharmacological management of children with persistent pain

Editorial | version published: 26 June 2019

https://doi.org/10.1002/14651858.ED000135

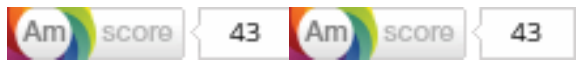

\title{
Neil O'Connell
}

View authors' declarations of interest

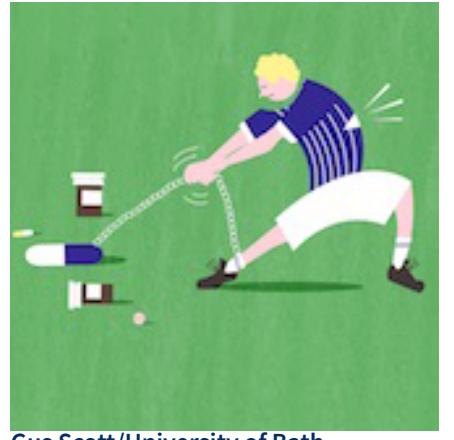

Gus Scott/University of Bath
For evidence-based practice to succeed we need a body of evidence that is large enough to offer precision, rigorous enough to inspire confidence, and direct enough to equitably represent all social groups. Yet in too many areas of clinical practice this remains an aspiration.

In their landmark overview of reviews, Eccleston and colleagues present us with an arresting example of this problem.[1] Persistent pain in children is common and results from a multitude of causes. It is responsible for

substantial suffering, disability, and costs to the child, their family, and to broader society.

Pharmacological interventions are often first-line approaches to pain treatment in children. If there are pharmacological treatment options that offer a good chance of benefitting children and adolescents with pain we need to know this so that we can offer those options with confidence. And if some of the options that are available in adults are not effective in children and adolescents or have an unfavourable safety profile then we need to know so that we can minimize the risks that we expose them to. A clear and upto-date overview of the evidence for their effectiveness is vital and timely.

We should find the results of this overview shocking. From 23 included systematic reviews across all pharmacological agents there were just six randomized controlled trials (RCTs) that involved children with chronic pain. Those RCTs provide only very low-quality evidence for any given drug option. Not a single trial was found in children with cancer-related chronic pain.

Before we shrug at the familiar and disappointing mantras of 'insufficient evidence' and 'more studies are required' it is useful to think more deeply about what these results mean, how this situation has arisen, and what it might take to meet our aspirations of evidence-based practice in paediatric persistent pain. In their discussion, Eccleston and colleagues offer us food for thought. 
These findings reflect the uncomfortable truth that evidence-based practice relating to drug options is not currently possible for clinicians or for children in persistent pain and their families. In comparison to the much more substantive and definitive evidence base available in adult care, the results point to a lack of equity in the research culture of pain management, discriminating against children and adolescents. They also highlight the considerable risks of suboptimal and unsafe pain management that this population is exposed to, potentially in perpetuity.

Eccleston and colleagues outline how we might have arrived here. Research in children is clearly challenging for a number of practical and ethical reasons, and recruiting large groups is often not possible. There is little market incentive for industry to invest and commit to large programmes of research in this patient group, particularly for existing medicines. We have not yet seen the level of sustained and substantial government or non-industry funding that might be needed to make a meaningful impact.

Expedience and the lack of a restrictive regulatory framework has facilitated the extrapolation of results from trials in adults to the management of children and the common off-license use in children of drugs licensed for adults. We should consider whether we are comfortable with that corner being cut. The assumptions of equivalent effectiveness and safety that underlie these practices are untested and have been challenged.[2] Still, more and larger trials will only go so far in addressing the safety concerns that this raises. There is currently little in the way of co-ordinated infrastructure to allow for effective pharmacovigilance relating to analgesic use in children.

So to re-use the bland cliché, more research is required. And urgently. The challenge is not insurmountable. While by no means perfect, a more substantive evidence base exists to guide the drug treatment of depression in children and adolescents.[3, 4] Judging by the rate of publications of new trials seen in the chronic pain overview, the ball has not begun to roll.

Eccleston and colleagues highlight the need not just for more trials, but for research that seeks to better understand the specific challenges of and barriers to producing quality evidence in paediatric pain. They offer ideas for exploring innovative alternatives to traditional RCTs, such as single case designs. They make the urgent case for national or trans-national registries of children and adolescents treated for chronic pain, with a primary focus on analgesic medicines.

Solving this problem may not require any great methodological innovations, just the will and resource to do well what has not yet been done. There are almost no RCTs of analgesic medicines for children with persistent pain due to cancer or any other cause, and that fact fails these children, and all involved in their care.

\section{Information}

\section{DOI:}


https://doi.org/10.1002/14651858.ED000135 scolaris.information.information.copy.clipboard (A) Check for updates

\section{Database:}

Cochrane Database of Systematic Reviews

\section{Published:}

26 June 2019

\section{Copyright:}

Copyright (c) 2019 The Cochrane Collaboration. Published by John Wiley \& Sons, Ltd.

\section{Article Metrics}

\section{Altmetric:}

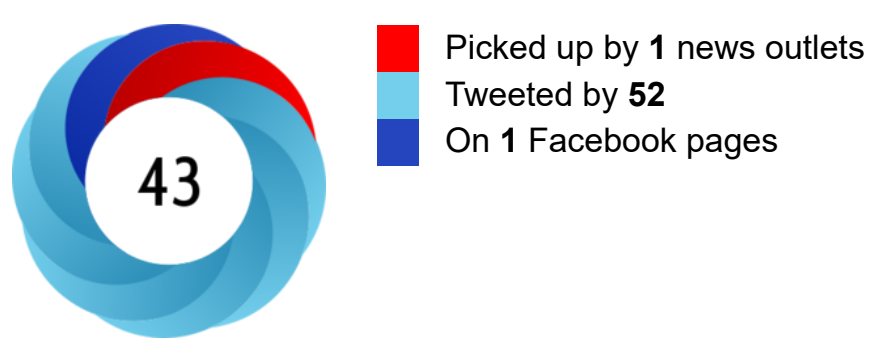

See more details

\section{Authors}

\section{Neil O'Connell}

Department of Clinical Sciences, Brunel University, London, UK neil.oconnell@brunel.ac.uk

Q More by this author on the Cochrane Library

\section{Declarations of interest}

The author has completed the ICMJE form for disclosure of potential conflicts of interest and has no conflicts to declare. 


\section{Provenance and peer review}

This editorial was commissioned based on a proposal by Cochrane Pain \& Anaesthesia and was not externally peer reviewed.

\section{References}

1. Ecccleston C, Fisher E, Cooper T, Grégoire MC, Heathcote LC, Krane E, et al. Pharmacological interventions for chronic pain in children and adolescents: an overview of systematic reviews. Pain 2019;June 19. https://doi.org/10.1097/j.pain.0000000000001609

2. Schechter NL, Walco GA. The potential impact on children of the CDC Guideline for Prescribing Opioids for Chronic Pain: above all, do no harm. JAMA Pediatrics2016;170(5):425-6. https://doi.org/10.1001/jamapediatrics.2016.0504

3. Hazell P, Mirzaie M. Tricyclic drugs for depression in children and adolescents. Cochrane Database of Systematic Reviews2013;(6):CD002317. https://doi.org/10.1002/14651858.CD002317.pub2

4. Hetrick SE, McKenzie JE, Cox GR, Simmons MB, Merry SN. Newer generation antidepressants for depressive disorders in children and adolescents. Cochrane Database of Systematic Reviews2012; (11):CD004851. https://doi.org/10.1002/14651858.CD004851.pub3 